\title{
Existence of the Exponentially Localised Wannier Functions
}

\author{
G. Nenciu* \\ Laboratory of Theoretical Physics, Joint Institute for Nuclear Research, Dubna SU-141980, USSR
}

\begin{abstract}
A partial answer (Theorem 1 below) to a problem concerning analytic and periodic families of projections in Hilbert spaces is given. As a consequence the existence of exponentially localised Wannier functions corresponding to nondegenerated bands of arbitrary three-dimensional crystals is proved.
\end{abstract}

\section{Statement of the Problem and the Result}

The present note is motivated by one of the few basic questions of the quantum theory of periodic solids in the one-electron approximation which is not completely solved: the existence of exponentially localised Wannier functions $[1,2]$. We shall consider only nondegenerated bands and neglect the spin (for the results obtained so far for degenerated bands, we refer to [3-5]). The Wannier functions falling off exponentially at infinity for arbitrary $n$-dimensional crystals. The proof is based on dimensional crystals $[4,6]$; ii) $n$-dimensional crystals $(n>1)$ with a center of inversion [4]; iii) arbitrary crystals (i.e. with or without a centre of inversion) in the tight binding limit $[4]^{1}$.

The result of this note is the existence of Wannier functions falling off exponentially at infinit for arbitrary $n$-dimensional crystals. The proof is based on a partial answer (Theorem 1 below) to the following problem concerning analytic families of projections in Hilbert spaces.

Problem P. Let $\mathscr{K}$ be a separable Hilbert space, $q$ be a positive integer, $\mathscr{I}_{a}^{q}=\left\{\mathbf{z}^{q}\right.$ $\left.=\left(z_{1}, \ldots, z_{q}\right) \in \mathbb{C}^{q}|| \operatorname{Im} z_{i} \mid<a, a>0\right\}$ and $Q\left(\mathbf{z}^{q}\right): \mathscr{K} \rightarrow \mathscr{K}$ be a projection-valued func-

* Permanent address: Central Institute of Physics, P.O. Box MG 6, Bucharest, Romania

1 There is a widespread opinion (see e.g. [2]) that the existence of exponentially localised Wannier functions has been proved for arbitrary $n$-dimensional crystals by Blount [7]. Unfortunately, this is not true since by his argument Blount proved only analyticity (as functions of the crystal momentum) of the Bloch functions, while for the exponential falling off the Wannier functions analyticity and periodicity of the Bloch functions is nedeed 
tion, analytic in $\mathscr{I}_{a}^{q}$ and satisfying:

$$
\begin{gathered}
Q\left(\mathbf{z}^{q}\right)=Q^{*}\left(\mathbf{z}^{q}\right), \mathbf{z}^{q} \in \mathbb{R}^{q}, \\
Q\left(\mathbf{z}^{q}\right)=Q\left(\mathbf{z}^{q}+2 \pi \mathbf{p}^{q}\right), \mathbf{z}^{q} \in \mathscr{I}_{a}^{q}, \mathbf{p}^{q}=\left(p_{1}, \ldots, p_{q}\right) \in \mathbb{Z}^{q} .
\end{gathered}
$$

Find a bounded, with bounded inverse, operator-valued function $A\left(\mathbf{z}^{q}\right): \mathscr{K} \rightarrow \mathscr{K}$, analytic in $\mathscr{I}_{a}^{q}$ and satisfying

$$
\begin{gathered}
Q\left(\mathbf{z}^{q}\right)=A\left(\mathbf{z}^{q}\right) Q(0) A^{-1}\left(\mathbf{z}^{q}\right), A(0)=1, \mathbf{z}^{q} \in \mathscr{I}_{a}^{q}, \\
A^{-1}\left(\mathbf{z}^{q}\right)=A^{*}\left(\mathbf{z}^{q}\right), \mathbf{z}^{q} \in \mathbb{R}^{q}, \\
A\left(\mathbf{z}^{q}\right) Q(0)=A\left(\mathbf{z}^{q}+2 \pi \mathbf{p}^{q}\right) Q(0), \mathbf{z}^{q} \in \mathscr{I}_{a}^{q}, \mathbf{p}^{q} \in \mathbb{Z}^{q} .
\end{gathered}
$$

Without the periodicity conditions (1.2), (1.5) the Problem $\mathrm{P}$ has been thoroughly investigated in connection with perturbation theory $[8,9]$, asymptotic theory of differential equations $[10,11]$, etc., and it is fully solved. For $q=1$ Problem $\mathrm{P}$ has been solved in [5] (see also [12] for finite dimensional $\mathscr{K}$ ). For $q>1$, except for the case $\left\|Q\left(\mathbf{z}^{q}\right)-Q(0)\right\|<1$, where a solution of Problem $\mathrm{P}$ can be constructed using the Sz-Nagy lemma on unitary equivalence of projections in Hilbert spaces (see e.g. [8, Chap. II.4]), we are not aware of any other results, and in fact it seems that in general Problem $\mathrm{P}$ does not admit solutions [13]. The result in Theorem 1 below gives another sufficient condition for the solutions of Problem $\mathrm{P}$ to exist.

Theorem 1. Under the conditions of Problem $P$, suppose

i) $\operatorname{dim} Q\left(\mathbf{z}^{q}\right)=1$.

ii) There exists an antilinear involution $\theta: \mathscr{K} \rightarrow \mathscr{K}$ such that

$$
\theta Q\left(\mathbf{z}^{q}\right) \theta=Q\left(-\mathbf{z}^{q}\right), \mathbf{z}^{q} \in \mathbb{R}^{q} .
$$

Then $A\left(\mathbf{z}^{q}\right)$ satisfying the requirements of Problem $P$ exists.

In the rest of this section, relying on Theorem 1, we shall outline the proof of the existence of exponentially localised Wannier functions (see also $[2,3,5]$ ). The Wannier functions, $\omega_{n}(\mathbf{x})$, are defined by (see e.g. [2, Sect. 5.1])

$$
\omega_{n}(\mathbf{x})=\text { const } \int_{B} \psi_{n, \mathbf{k}}(\mathbf{x}) d \mathbf{k},
$$

where $B$ is the Brillouin zone and $\psi_{n, \mathbf{k}}(\mathbf{x})=\exp (i \mathbf{k} \cdot \mathbf{x}) U_{n, \mathbf{k}}(\mathbf{x})$ are the Bloch functions of the considered band, $n$. The definition is not unique since $\psi_{n, \mathbf{k}}$ are defined by the eigenvalue problem only up to a $\mathbf{k}$ dependent phase factor. Rewriting (1.8) as

$\omega_{n}(\mathbf{x})=$ const $\int_{B} \exp \left(i \mathbf{R}_{\mathbf{x}} \cdot k\right) \exp \left(i \varrho_{\mathbf{x}} \cdot k\right) U_{n, \mathbf{k}}(\mathbf{x}) d \mathbf{k}, \mathbf{x}=\varrho_{\mathbf{x}}+\mathbf{R}_{\mathbf{x}}$, where $\varrho_{\mathbf{x}}$ belongs to the unit cell and $\mathbf{R}_{\mathbf{x}}$ is a vector of the direct lattice, the Paley-Wiener theorem implies [4] that the existence of exponentially localised Wannier functions is equivalent to the existence of Bloch functions $\tilde{\psi}_{n, \mathbf{k}}$ for which $\tilde{v}_{n, \mathbf{k}}(\mathbf{x}) \equiv \exp \left(i \varrho_{\mathbf{x}} \cdot \mathbf{k}\right) \tilde{U}_{n, \mathbf{k}}(\mathbf{x})$ is analytic and periodic in $\mathbf{k}$. 
Let $Q_{n}(\mathbf{k})$ be the orthogonal projection in $L^{2}$ (unit cell) corresponding to $v_{n, \mathbf{k}}$ [note that in $Q_{n}(\mathbf{k})$ the arbitrary phase factor cancels out]. We claim that $Q_{n}(\mathbf{k})$ is the restriction to $B$ of a projection-valued function satisfying the requirements of Theorem 1 . The analyticity follows from the fact that $u_{n, \mathbf{k}}$ can be chosen to be analytic by $\mathbf{k}-\mathbf{p}$ perturbation theory $[7,9]$. The periodicity follows from the fact that $\psi_{n, \mathbf{k}}$ and then $v_{n, \mathbf{k}}$ are periodic in $\mathbf{k}$ up to a phase factor. If $\theta$ is the involution representing the complex conjugation (i.e. time reversal in a theory in which spin is neglected), then $\theta \psi_{n, \mathbf{k}}$ equals $\psi_{n,-\mathbf{k}}$ [14] up to a phase factor, wherefrom (1.7) follows. Finally $\operatorname{dim} Q_{n}(\mathbf{k})=1$ from the nondegeneracy of the considered band. Let now the phase factor for $v_{n, 0}$ be fixed and $\tilde{v}_{n, \mathbf{k}}=A(\mathbf{k}) v_{n, 0}$, where $A(\mathbf{k})$ is given by Theorem 1 applied to $Q_{n}(\mathbf{k})$. Clearly $\tilde{v}_{n, \mathbf{k}}$ is analytic and periodic in $\mathbf{k}$.

\section{Proof of Theorem 1}

We shall construct $A_{i}\left(\mathbf{z}^{i}\right), \mathbf{z}^{i} \in \mathbb{C}^{i}, i=1, \ldots, q$ analytic in $\mathscr{I}_{a}^{i}$, unitary for $\mathbf{z}^{i} \in \mathbb{R}^{i}$ and satisfying

$$
\begin{gathered}
A_{i}\left(\mathbf{z}^{i}\right) Q\left(\mathbf{z}^{i-1}, 0\right) A_{i}^{-1}\left(\mathbf{z}^{i}\right)=Q\left(\mathbf{z}^{i}, 0\right), \quad A_{i}(0)=1, \\
A_{i}\left(\mathbf{z}^{i}\right) Q\left(\mathbf{z}^{i-1}, 0\right)=A_{i}\left(\mathbf{z}^{i}+2 \pi \mathbf{p}^{i}\right) Q\left(\mathbf{z}^{i-1}, 0\right), \quad \mathbf{p}^{i} \in \mathbb{Z}^{i} .
\end{gathered}
$$

Clearly

$$
A\left(\mathbf{z}^{q}\right)=\prod_{i=0}^{q-1} A_{q-i}\left(\mathbf{z}^{q-i}\right)
$$

satisfies all the requirements of the theorem. We shall give in detail the construction of $A_{q}\left(\mathbf{z}^{q}\right)$, the construction of $A_{i}\left(\mathbf{z}^{i}\right), i=1, \ldots, q-1$ being similar.

Let $\tilde{A}_{q}\left(\mathbf{z}^{q}\right)$ be the solution of

$$
i \frac{d}{d z_{q}} \tilde{A}_{q}\left(\mathbf{z}^{q}\right)=i\left(\left(1-2 Q\left(\mathbf{z}^{q}\right)\right) \frac{d}{d z_{q}} Q\left(\mathbf{z}^{q}\right)\right) \tilde{A}_{q}\left(\mathbf{z}^{q}\right), \tilde{A}_{q}\left(\mathbf{z}^{q-1}, 0\right)=1 .
$$

Then [8, Chap. $2.4 ; 9$, Chap. XII.2;11], $\tilde{A}_{q}\left(\mathbf{z}^{q}\right)$ is analytic in $\mathscr{I}_{a}^{q}$, unitary for $\mathbf{z}^{q} \in \mathbb{R}^{q}$, satisfies (2.1), (2.2) with respect to $\mathbf{z}^{q-1}$ but not (2.2) with respect $z_{q}$. Consider

$$
T\left(\mathbf{z}^{q-1}\right)=\tilde{A}_{q}\left(\mathbf{z}^{q-1}, \pi\right) \tilde{A}_{q}^{-1}\left(\mathbf{z}^{q-1},-\pi\right) .
$$

Clearly $T\left(\mathbf{z}^{q-1}\right)$ is analytic invertible and periodic in $\mathscr{I}_{a}^{q-1}$. Supposing that $T\left(\mathbf{z}^{q-1}\right)$ has a logarithm analytic and periodic in $\mathscr{I}_{a}^{q-1}$ [i.e. $T\left(\mathbf{z}^{q-1}\right)=\exp \left(2 \pi i M\left(\mathbf{z}^{q-1}\right)\right.$, $M\left(\mathbf{z}^{q-1}\right)$ analytic and periodic in $\mathscr{I}_{a}^{q-1}$, then the arguments in [5] show that $\exp \left(i z_{q} M\left(\mathbf{z}^{q-1}\right)\right) \tilde{A}_{q}\left(\mathbf{z}^{q}\right)$ satisfies all the requirements for $A_{q}\left(\mathbf{z}^{q}\right)$ with (2.2) replaced by the following (stronger) condition:

$$
\exp \left(i z_{q} M\left(\mathbf{z}^{q-1}\right)\right) \tilde{A}_{q}\left(\mathbf{z}^{q}\right)=\exp \left(i\left(z_{q}+2 \pi p_{q}\right) M\left(\mathbf{z}^{q-1}+2 \pi \mathbf{p}^{q-1}\right)\right) \tilde{A}_{q}\left(\mathbf{z}^{q}+2 \pi \mathbf{p}^{q}\right) .
$$

Unfortunately, we have been unable to prove that $T\left(\mathbf{z}^{q-1}\right)$ admits an analytic and periodic logarithm [in fact for $\mathbf{z}^{q-1} \notin \mathbb{R}^{q-1}$, we do not know, if $T\left(\mathbf{z}^{q-1}\right)$ admits a logarithm at all], and therefore we shall follow a slightly different route, suggested 
by the fact that the weaker condition (2.2) instead of (2.6) is required. Due (1.2) and to the fact that $\tilde{A}_{q}\left(\mathbf{z}^{q}\right)$ satisfies (2.1), we have

$$
\left[T\left(\mathbf{z}^{q-1}\right), Q\left(\mathbf{z}^{q-1}, \pi\right)\right]=0,
$$

i.e. for $f_{1} \in Q\left(\mathbf{z}^{q-1}, \pi\right) \mathscr{K}$,

$$
T\left(\mathbf{z}^{q-1}\right) f_{1}=\lambda\left(\mathbf{z}^{q-1}\right) f_{1},
$$

where [recall that $\left.\operatorname{dim} Q\left(\mathbf{z}^{q}\right)=1\right] \lambda\left(\mathbf{z}^{q-1}\right)$ is a complex-valued function. We shall prove [and it is just the point where (1.7) is used] that there exists a unique function $\phi\left(\mathbf{z}^{q-1}\right)$ analytic and periodic in $\mathscr{I}_{a}^{q-1}$, real for $\mathbf{z}^{q-1} \in \mathbb{R}^{q-1}, \phi(0) \in[0,1)$ such that

$$
\lambda\left(\mathbf{z}^{q-1}\right)=\exp \left(2 \pi i \phi\left(\mathbf{z}^{q-1}\right)\right)
$$

Taking this for granted it is easy to finish the proof of Theorem 1 with

$$
A_{q}\left(\mathbf{z}^{q}\right) \equiv \exp \left(-i z_{q} \phi\left(\mathbf{z}^{q-1}\right)\right) \tilde{A}_{q}\left(\mathbf{z}^{q}\right) .
$$

Indeed all the required properties except for the periodicity in $z_{q}$ are obvious. Since $A_{q}\left(\mathbf{z}^{q}\right)$ as defined by (2.9) satisfies a differential equation with periodic coefficients, the only thing we have to verify is that

$$
A_{q}\left(\mathbf{z}^{q-1},-\pi\right) Q\left(\mathbf{z}^{q-1}, 0\right)=A_{q}\left(\mathbf{z}^{q-1}, \pi\right) Q\left(\mathbf{z}^{q-1}, 0\right) .
$$

Now (2.10) can be verified as follows using (1.2), (2.2), (2.5), $\left[T\left(\mathbf{z}^{q-1}\right), Q\left(\mathbf{z}^{q-1}, \pi\right)\right]=0$, (2.7) and (2.8),

$$
\begin{aligned}
& A_{q}\left(\mathbf{z}^{q-1},-\pi\right) Q\left(\mathbf{z}^{q-1}, 0\right)=\exp \left(i \pi \phi\left(\mathbf{z}^{q-1}\right)\right) T^{-1}\left(\mathbf{z}^{q-1}\right) \tilde{A}_{q}\left(\mathbf{z}^{q-1}, \pi\right) \\
& \cdot Q\left(\mathbf{z}^{q-1}, 0\right)=\exp \left(i \pi \phi\left(\mathbf{z}^{q-1}\right)\right) T^{-1}\left(\mathbf{z}^{q-1}\right) Q\left(\mathbf{z}^{q-1}, \pi\right) \tilde{A}_{q}\left(\mathbf{z}^{q-1}, \pi\right) \\
& \quad=A_{q}\left(\mathbf{z}^{q-1}, \pi\right) Q\left(\mathbf{z}^{q-1}, 0\right) .
\end{aligned}
$$

Let us return to (2.8). The invertibility of $T\left(\mathbf{z}^{q-1}\right)$ implies $\lambda\left(\mathbf{z}^{q-1}\right) \neq 0$ for $\mathbf{z}^{q-1} \in \mathscr{I}_{a}^{q-1}$. Let $0=f \in Q(0, \pi)$. There exists a neighbourhood $X$ of 0 in $\mathbb{C}^{q-1}$ such that $\left(f, Q\left(\mathbf{z}^{q-1}, \pi\right) f\right) \neq 0$. Then

$$
\lambda\left(\mathbf{z}^{q-1}\right)=\left(f, Q\left(\mathbf{z}^{q-1}, \pi\right) f\right)^{-1}\left(f, Q\left(\mathbf{z}^{q-1}, \pi\right) T\left(\mathbf{z}^{q-1}\right) f\right),
$$

and hence $\lambda\left(\mathbf{z}^{q-1}\right)$ is analytic in $X$. By an analytic continuation argument $\lambda\left(\mathbf{z}^{q-1}\right)$ is analytic in $\mathscr{I}_{a}^{q-1}$. Hence (2.8) holds true with $\phi\left(z^{q-1}\right)$ analytic in $\mathscr{I}_{a}^{q-1}$. The reality of $\phi\left(\mathbf{z}^{q-1}\right)$ for $\mathbf{z}^{q-1} \in \mathbb{R}^{q-1}$ follows from the unitarity of $T\left(\mathbf{z}^{q-1}\right)$. The periodicity of $\lambda\left(\mathbf{z}^{q-1}\right)$ is obvious. Since $\frac{d \lambda}{d z_{e}}=i \lambda \frac{d \phi}{d z_{e}}, \frac{d \phi}{d z_{e}}$ are periodic, and hence

$$
\phi\left(\mathbf{z}^{q-1}\right)=\psi\left(\mathbf{z}^{q-1}\right)+\sum_{l=1}^{q-1} p_{e} z_{e},
$$

where $\psi\left(\mathbf{z}^{q-1}\right)$ is periodic and $p_{e}$ are integers. We shall show now that (1.7) implies

$$
\phi\left(\mathbf{z}^{q-1}\right)=\phi\left(-\mathbf{z}^{q-1}\right) .
$$


From (1.7) and (2.4) it follows :

$$
i \frac{d}{d z_{q}} \theta \tilde{A}_{q}\left(-\mathbf{z}^{q}\right) \theta=i\left(\left(1-2 Q\left(\mathbf{z}^{q}\right)\right) \frac{d}{d z_{q}} Q\left(\mathbf{z}^{q}\right)\right) \theta \tilde{A}_{q}\left(-\mathbf{z}^{q}\right) \theta,
$$

where of

$$
\theta \tilde{A}_{q}\left(-\mathbf{z}^{q}\right) \theta=\tilde{A}_{q}\left(\mathbf{z}^{q}\right), \quad \mathbf{z}^{q} \in \mathbb{R}^{q},
$$

and hence

$$
\theta T\left(-\mathbf{z}^{q-1}\right) \theta=T^{-1}\left(\mathbf{z}^{q-1}\right), \quad \mathbf{z}^{q-1} \in \mathbb{R}^{q-1} .
$$

From (1.2) and (1.7), one has

$$
\theta Q\left(\mathbf{z}^{q-1}, \pi\right) \theta=Q\left(-\mathbf{z}^{q-1}, \pi\right) .
$$

Let now $f \in Q\left(-\mathbf{z}^{q-1}, \pi\right) \mathscr{K}$. Using (2.14), (2.15) and the reality of $\phi$, one has

$$
\begin{aligned}
\theta T\left(\mathbf{z}^{q-1}\right) \theta f & =\exp \left(-2 \pi i \phi\left(-\mathbf{z}^{q-1}\right)\right) f \\
& =\exp \left(-2 \pi i \phi\left(\mathbf{z}^{q-1}\right)\right) f
\end{aligned}
$$

which proves (2.12). Now (2.11) implies $p_{e}=0$ in (2.11). Indeed, for example

$$
\phi(-\pi, 0, \ldots, 0)=\psi(-\pi, 0, \ldots, 0)-p_{1} \pi=\psi(\pi, 0, \ldots, 0)+p_{1} \pi,
$$

and the periodicity of $\psi$ implies $p_{1}=0$. The proof of the theorem is completed.

\section{References}

1. Wannier, G.H.: The structure of electronic excitation levels in insulating crystals. Phys. Rev. 52, 191-197 (1937)

2. Callaway, J.: Quantum theory of the solid state. New York: Academic Press 1974

3. Des Cloizeaux, J.: Energy bands and projection operators in a crystal: analytic and asymptotic properties. Phys. Rev. 135 A, 685-697 (1964)

4. Des Cloizeaux, J.: Analytical properties of $n$-dimensional energy bands and Wannier functions. Phys. Rev. 135 A, 698-707 (1964)

5. Nenciu, A., Nenciu, G. : Dynamics of Bloch electrons in external electric fields: II. The existence of Stark-Wannier ladder resonances. J. Phys. A 15, 3313-3328 (1982)

6. Kohn, W.: Analytic properties of Bloch waves and Wannier functions. Phys. Rev. 115, 809-821 (1958)

7. Blount, I.E.: Solid State Phys. 13, 305-373 (1962)

8. Kato, T.: Perturbation theory of linear operators. Berlin, Heidelberg, New York: Springer 1966

9. Reed, M., Simon, B.: Methods of modern mathematical physics, Vol. IV. New York, San Francisco, London: Academic Press 1978

10. Krein, S.G.: Linear differential equations in Banach spaces. Moskva: Nauka 1967

11. Wasow, W.: Topics in the theory of linear ordinary differential equations having singularities with respect to a parameter. Lecture Notes, IRMA Univ. Louis Pasteur, Strasbourg 1977

12. Sibuya, Y.: Math. Ann. 161, 67-77 (1965)

13. Hsieh, P-F., Sibuya, Y.: J. Math. Anal. Appl. 14, 332-340 (1966)

14. Kittel, C.: Quantum theory of solids. New York: Wiley 1963

Communicated by B. Simon

Received October 27, 1982; in received form May 6, 1983 
